\title{
Emission Spectra from Internal Shocks in Gamma- Ray Burst Sources
}

\section{Citation}

Pilla, Ravi P., and Abraham Loeb. 1998. "Emission Spectra from Internal Shocks in Gamma-Ray Burst Sources." The Astrophysical Journal 494 (2): L167-71. https://doi.org/10.1086/311193.

\section{Permanent link}

http://nrs.harvard.edu/urn-3:HUL.InstRepos:41393216

\section{Terms of Use}

This article was downloaded from Harvard University's DASH repository, and is made available under the terms and conditions applicable to Other Posted Material, as set forth at http:// nrs.harvard.edu/urn-3:HUL.InstRepos:dash.current.terms-of-use\#LAA

\section{Share Your Story}

The Harvard community has made this article openly available.

Please share how this access benefits you. Submit a story.

\section{Accessibility}




\title{
Emission Spectra from Internal Shocks in Gamma-Ray-Burst Sources
}

\author{
Ravi P. Pilla \\ Department of Physics, Columbia University, 538 W 120th street, New York, 10027, NY; \\ ravi@cuphyb.phys.columbia.edu
}

Abraham Loeb

Astronomy Department, Harvard University, 60 Garden Street, Cambridge, MA 02138;

aloeb@cfa.harvard.edu

\begin{abstract}
Unsteady activity of $\gamma$-ray burst sources leads to internal shocks in their emergent relativistic wind. We study the emission spectra from such shocks, assuming that they produce a power-law distribution of relativistic electrons and posses strong magnetic fields. The synchrotron radiation emitted by the accelerated electrons is Compton up-scattered multiple times by the same electrons. A substantial component of the scattered photons acquires high energies and produces $e^{+} e^{-}$pairs. The pairs transfer back their kinetic energy to the radiation through Compton scattering. The generic spectral signature from pair creation and multiple Compton scattering is highly sensitive to the radius at which the shock dissipation takes place and to the Lorentz factor of the wind. The entire emission spectrum extends over a wide range of photon energies, from the optical regime up to $\mathrm{TeV}$ energies. For reasonable values of the wind parameters, the calculated spectrum is found to be in good agreement with the burst spectra observed by BATSE.
\end{abstract}

Subject headings: gamma rays: bursts - radiation mechanisms: non-thermal submitted to ApJ Letters, October 1997

\section{Introduction}

The detection of FeII and MgII absorption lines at a redshift of $z=0.835$ in the optical spectrum of GB970508 (Metzger et al. 1997), provided the first confirmation that $\gamma$-ray bursts (GRBs) originate at cosmological distances. Most of the qualitative properties of cosmological GRBs are explained by the fireball model (see e.g., Goodman 1986; Paczyński 1986; Mészáros \& 
Rees $1993[\mathrm{MR}])$. In this model, a compact $\left(\sim 10^{6-7} \mathrm{~cm}\right)$ source releases an energy of $\sim 10^{52} \mathrm{erg}$ over a duration $t_{d} \lesssim 10^{2}$ seconds with a negligible baryonic contamination $\left(\lesssim 10^{-5} M_{\odot}\right)$. Unsteady activity of the source results in a wind composed of many thin layers (fireball shells) of varying energy and baryonic mass. Within each shell the high energy-density at the source results in an optically thick $e^{+} e^{-}$-pair plasma that expands and accelerates to relativistic speeds. After an initial acceleration phase, the radiation and thermal energy of the fireball plasma is converted into the kinetic energy associated with the radial motion of the protons. Collisions between the shells can convert part of that kinetic energy into radiation and yield the primary GRB via synchrotron emission and inverse-Compton scattering (Paczyński \& Xu 1994; Rees \& Mészáros 1994 [RM]; Sari \& Piran 1997 [SP]). As the wind continues to expand, it impinges on the surrounding medium and eventually drives a relativistic blastwave in it, which heats fresh gas and accelerates electrons to relativistic speeds, thus producing the delayed afterglow radiation observed on time scales of hours to months (van Paradijs et al. 1997; Bond 1997; Djorgovski et al. 1997; Mignoli et al. 1997; Frail et al. 1997) via synchrotron emission (Wijers, Rees, \& Mészáros 1997;Waxman 1997a,b; Vietri 1997a,b) .

The primary GRB emission is more likely caused by internal shocks than the external shocks (MR), since they can occur closer to the source and thus account for the rapid variability observed in many bursts (RM; SP). Unsteady activity of the central source naturally results in faster shells overtaking slower ones in front of them, and hence in energy dissipation by internal shocks. The complex temporal structure observed in GRBs then reflects the activity-history of their sources (SP; Kobayashi, Piran, \& Sari 1997 [KPS]).

It is often assumed that behind internal shocks, electrons are Fermi accelerated with a near equipartition energy density and magnetic fields acquire nearly equipartition strength. The electrons cool by synchrotron emission and inverse-Compton (IC) scattering off the synchrotron photons. Under typical conditions, the time scale for IC scattering is shorter than the synchrotron cooling time. Multiple scattering of the photons boosts a significant fraction of the radiation energy to frequencies above the $e^{+} e^{-}$-pair creation threshold. The pairs produced in this process are also relativistic and cool rapidly by IC scattering. Since the annihilation time of these pairs is longer than the hydrodynamic time in the comoving frame, they survive in the wind for a long time. Although the creation of pairs and their subsequent cooling is likely to leave noticeable imprints on the emergent radiation spectrum, it has not been analyzed before in the GRB literature. Since the photon and electron densities decline rapidly with radius, the strength of these signatures can serve as a probe of the radius at which the internal shocks occur.

The GRB spectrum should also depend on the level of baryonic contamination in the wind. The extreme limit of pure energy release with no baryons was ruled out in the past based on the prediction that a point explosion of this type would lead to a roughly thermal spectrum (Goodman 1986). One can place a lower limit on the baryonic mass in the fireball shells by requiring that internal shocks should occur before an external shock does (this limit depends on the ambient medium density). On the other hand, an upper limit can be placed based on the variability time 
scale of the source and the condition that the shells be optically thin at the radius where internal shocks occur.

In this Letter we study in detail the emergent spectra from the collision of two fireball shells: In particular, we quantify the significance of the radiation processes which were previously ignored in the literature, such as multiple Compton scattering, $\mathrm{e}^{+} \mathrm{e}^{-}$creation, and their subsequent cooling. We use the collision kernels, reaction rates, and the computational techniques given in Pilla \& Shaham (1997 [PS]). More details about this calculation and an elaborate study of the spectral characteristics of relativistic shocks will be included in a subsequent publication (Pilla \& Loeb 1997). In $\S 2$ we describe our model and specify the physical conditions in the emission region. In $\S 3$ we outline the relevant radiation processes and compute the model spectra. Finally, $\S 4$ summarizes the main implications of this work.

\section{Physical Properties of Internal Shocks}

The typical fireball dynamics (Piran, Shemi, \& Narayan 1993, [PSN]; Mészáros, Laguna, \& Rees 1993; Sari, Narayan, \& Piran 1996 [SNP]; SP; and KPS) can be illustrated by considering a single shell of total energy $E=10^{51} E_{51} \mathrm{erg}$, rest mass $M=10^{27} M_{27} \mathrm{~g}$, and initial radius $r_{0}=10^{7} r_{7} \mathrm{~cm}$. After a brief acceleration phase, the Lorentz factor of the shell reaches a constant value $\Gamma$ at an observer-frame radius $r_{m} \approx \Gamma r_{0}$ (the protons are taken to be non-relativistic in the comoving frame, before the collision of shells). The energy of the shell is predominantly kinetic beyond this stage. Outside the radius $r \approx r_{\text {exp }}=2 \Gamma^{2} r_{0}$ (all radii in the present analysis are measured in the observer's frame), the comoving width of the shell increases linearly with radius (PSN). The comoving proton density scales as $n \propto r^{-2}$ for shell radii $r \leq r_{\text {exp }}$ and as $n \propto r^{-3}$ for $r>r_{\text {exp }}$. The Thomson optical depth of the shell is $\tau \approx\left(r_{t} / r\right)^{2}$ for $r \geq r_{\text {exp }}$, where $r_{t} \approx\left(M \sigma_{T} / 4 \pi m_{p}\right)^{1 / 2} \approx 6.3 \times 10^{12} M_{27}^{1 / 2} \mathrm{~cm}$ is the radius at which the shell becomes optically thin to Thomson scattering by its own electrons. Here $\sigma_{T}$ is Thomson cross section and $m_{p}$ is the proton rest mass. For $r>r_{\text {exp }}$, the comoving width of the shell is $\delta \approx r / 2 \Gamma$. Two photons which are emitted with a proper-time difference $\sim \delta / c$, reach the observer with a time separation $\sim \delta / 2 \Gamma c \approx r / 4 \Gamma^{2} c(\mathrm{SP})$. Assuming that the radiative cooling time is much shorter than the light transit time through the system, one finds that the observed width of the radiation pulse $t_{p}$ and the radius of the emission region $r_{e}$ are related through $r_{e} \approx 2 \Gamma^{2} c t_{p}$, which in turn can be used to constrain the values of $\delta, \tau$, and $n$ from observations. The emission spectrum will be nonthermal only if $r_{e} \gg r_{t}$.

Now consider a wind of total duration $t_{d}$, composed of many thin fireball shells of thickness

\footnotetext{
*In principle, each individual peak in the burst light-curve might correspond to a collision of a pair of shells (KPS). The time-averaged flux of the entire burst is then an energy-weighted sum of the contributions from individual collisions. Here we provide the time-averaged spectra for the collision of two shells, whereas the generalization to the entire burst (with multiple peaks) is straightforward.
} 
$\delta \ll c t_{d}$ ( $\delta$ may change from one shell to another). We assume that for $r>r_{\text {exp }}$ there are regions in the wind where slower shells precede the faster ones, i.e. $d \Gamma(r) / d r<0$, where $\Gamma(r)$ is the local Lorentz factor. The spatial extent of the wind is typically $\sim c t_{d} \gg r_{0}$ (in fact, $\delta \gg r_{0}$ also holds in general). It was shown by Waxman \& Piran (1994) that under these conditions the wind layers are susceptible to Rayleigh-Taylor instability because a rarefied fluid shell is pushing against a denser one. The resulting turbulent mixing will complicate the shock structure and deform it away from a simple planar geometry. Merging of a rarefied shell with a denser one might therefore be accompanied by the formation of "fingers" perpendicular to the shell walls, similar to the non-relativistic shock structure in supernova remnants (e.g., Jun \& Norman 1996). The combined shells would then break into bubbles of different sizes, and the energy dissipation would take place near the bubble walls, due to collisions among them or instabilities on their surfaces (Kamionkowski \& Freese 1992).

By assuming that the shells as well as the shock fronts remain planar, KPS had found that the dissipation efficiency of internal shocks might obtain high values $(\sim 50 \%)$ for reasonable wind parameters. We assume that similar efficiencies are achieved in the case of unstable mixing. Since the combined area of the bubble walls greatly exceeds that of a planar shock, the electron acceleration efficiency in the present case is likely to be higher. However the temporal and spectral characteristics of the bursts might be different in the two cases. For a planar shock the accelerated electrons populate a thin layer around the shock front since their cooling time is much shorter than the transit time of sound waves across the shells. However, in the unstable mixing case we assume, to a first approximation, that the energy dissipation takes place nearly uniformly and simultaneously throughout the entire volume of the emission region. For the purpose of estimating the physical conditions involved, we take $E \approx 10^{51} \mathrm{erg}, M \approx 10^{27} \mathrm{~g}, r_{e} \approx 10^{14} \mathrm{~cm}$, and a bulk Lorentz factor of the emission region of $\Gamma \approx 400$. These values yield $n \approx 3.1 \times 10^{10} \mathrm{~cm}^{-3}$, $\tau \approx 3 \times 10^{-3}$, a comoving width of the post-shock shell $\Delta \approx 1.4 \times 10^{11} \mathrm{~cm}$, and an average Lorentz factor of the protons in the comoving frame (i.e., after shock heating) of $\bar{\gamma}_{p} \approx 3$.

\section{Radiation Mechanisms and Spectra}

Acceleration of electrons to relativistic energies and the presence of strong magnetic fields are essential for converting the energy dissipated by the shock waves into radiation. Since the physics of neither of these processes is well understood, we parameterize the corresponding energy densities in units of their equipartition values. A magnetic equipartition parameter

$\zeta_{B}=B^{2} / 8 \pi n m_{p} c^{2}\left(\bar{\gamma}_{p}-1\right)$, corresponds to a field strength $B \approx 1.9\left[\zeta_{B} n_{10}\left(\bar{\gamma}_{p}-1\right)\right]^{1 / 2} 10^{4} \mathrm{G}$, where $n_{10}=n /\left(10^{10} \mathrm{~cm}^{-3}\right)$ (all quantities are comoving, unless stated otherwise). We assume that the electrons are accelerated throughout the emission region. The amount of energy transferred from protons to electrons is uncertain, and we define the acceleration efficiency $\zeta_{e}$ in such a way that the average Lorentz factor of the electrons immediately after they are heated (via Fermi-type acceleration) is $\bar{\gamma}_{0}=\zeta_{e}\left(\bar{\gamma}_{p}-1\right) m_{p} / m_{e}$, where $m_{e}$ is the electron rest mass. Because the Coulomb 
collision time $\sim 1 / c n \sigma_{T} \ln \Lambda \sim \Delta / c \tau \ln \Lambda \gg \Delta / c$, we can safely ignore collisional relaxation in our analysis (here $\ln \Lambda \sim 30$ is the Coulomb logarithm).

Collisionless acceleration of electrons can be efficient if they are tightly coupled to the protons and the magnetic field by means of plasma waves (Kirk 1994). The typical Alfvén speed in the plasma is $v_{A}=B /\left(4 \pi n m_{p}\right)^{1 / 2}=\min \left(1,1.4 \zeta_{B}^{1 / 2}\right) c$. The Larmor radius of an electron with a Lorentz factor $\gamma$ is $r_{L, e} \approx 0.1 \gamma\left(\zeta_{B} n_{10}\right)^{-1 / 2} \mathrm{~cm}$, and that of a proton of equal Lorentz factor, $r_{L, p}$, is larger by a factor $m_{p} / m_{e}$. The corresponding acceleration time scales (e.g., Hillas 1984) are, $t_{a c c, e} \sim c r_{L, e} / v_{A}^{2} \simeq 1.7 \times 10^{-13} \gamma\left(\zeta_{B}^{3} n_{10}\right)^{-1 / 2}$ sec and $t_{a c c, p} \sim\left(m_{p} / m_{e}\right) t_{a c c, e}$, respectively. Synchrotron losses limit the maximum value of the electron Lorentz factor to $\gamma_{\max , e} \sim 3.2 \times 10^{5}\left(n_{10}^{3} \zeta_{B}\right)^{-1 / 4}$. The minimum value is determined by $\bar{\gamma}_{0}$ and the shape of the electron distribution. We assume that the fraction of electrons per unit Lorentz factor $\gamma$ has the form $F_{e}(\gamma)=(p-1) \gamma_{\min }^{p-1} \gamma^{-p}$ for $\gamma_{\min } \leq \gamma \leq \gamma_{\max }$. Thus $\gamma_{\min }=(p-2) \bar{\gamma} /(p-1)$, where $\bar{\gamma}$ is the average Lorentz factor at any given time. The energy density in electrons immediately after their acceleration is $u_{0}=n m_{e} c^{2} \bar{\gamma}_{0}$. In our model the radiation time scale is much shorter than the hydrodynamic expansion time scale in the comoving frame (which leads to a radiative efficiency of nearly $100 \%$ ) and the radiation density at the end of electron cooling is therefore $u_{0}$. We compute the spectra for $\zeta_{B}=0.1, \zeta_{e}=0.3$, and $p=3.5$.

\section{Electron Cooling and Radiation Spectrum}

We assume that the radiation energy density is initially small, and hence the electrons start losing their energy via synchrotron emission. As the energy density of the emitted radiation builds up, cooling via IC scattering becomes important as well. The typical time scale for synchrotron or IC losses is $t_{c} \approx q / \lambda c n \sigma_{T} \bar{\gamma}^{2}$, where $\lambda=\zeta_{B} / \zeta_{e}$ in the synchrotron case and $\lambda=u_{\gamma} / u_{0}$ in the IC case. Here $u_{\gamma}$ is the radiation energy density and $q$ is a dimensionless constant of order unity. For typical GRB conditions $t_{a c c, e} \ll t_{c} \ll t_{0}$, where $t_{0} \approx \Delta / c$ is the light transit time through the system. Therefore electron cooling takes place only locally. We assume that the acceleration proceeds throughout the cooling phase in such a way that it maintains a steady power-law distribution with a constant index $p$, while $\bar{\gamma}$ declines due to radiative losses. If $\zeta_{B} \ll \zeta_{e}$, the synchrotron cooling time is long and multiple IC scatterings become important. Each scattering in the Thomson regime increases the photon energy by a factor $4\left\langle\gamma^{2}\right\rangle / 3$ (Loeb, McKee, \& Lahav 1991), so that some of the photons are eventually boosted into the Klein-Nishina (KN) regime. As $\bar{\gamma}$ decreases, the up-scattered part of the spectrum spreads over many decades in frequency. The electron energy density $u_{e}=n m_{e} c^{2} \bar{\gamma}$ changes at a rate

$$
\frac{d u_{e}}{d t}=\left(\frac{d u_{e}}{d t}\right)_{s y}+\left(\frac{d u_{e}}{d t}\right)_{I C}
$$

where $\left(d u_{e} / d t\right)_{s y}=-4 u_{B} / 3 T, u_{B}=B^{2} / 8 \pi, 1 / T=c n \sigma_{T}\left\langle\gamma^{2}\right\rangle_{0}$, and $\left\langle\gamma^{2}\right\rangle_{0}$ is the average value of $\gamma^{2}$ over the initial electron distribution. The IC cooling rate, $\left(d u_{e} / d t\right)_{I C}$, is derived below. The characteristic cooling time $t_{c}$ is a few times $T$ under typical conditions. If $u_{\gamma}(t)$ is the instantaneous energy density of radiation, then energy conservation implies $u_{\gamma}(t)+u_{e}(t)=u_{0}$ 
(which is constant), and by assumption $u_{\gamma}(0)=0$. The instantaneous radiation spectrum can be characterized by $\Phi(\varepsilon, t)$ so that $u_{\gamma}(t) \Phi(\varepsilon, t) d \varepsilon=v(\varepsilon, t) d \varepsilon$ is the fraction of the radiation energy density within an interval $d \varepsilon$ around $\varepsilon$; here $\varepsilon=h \nu / m_{e} c^{2}, \nu$ is the photon frequency, and $h$ is Planck's constant.

The spectral evolution rate (see Pilla \& Loeb 1997) is derived from the equation

$$
u_{\gamma}(t) \frac{\partial}{\partial t} \Phi(\varepsilon, t)-\Phi(\varepsilon, t) \frac{d u_{e}}{d t}=\left[\frac{\partial}{\partial t} v(\varepsilon, t)\right]_{s y}+\left[\frac{\partial}{\partial t} v(\varepsilon, t)\right]_{I C},
$$

where we have used the fact that $d u_{\gamma} / d t=-d u_{e} / d t$. The first term on the right hand side is the synchrotron emissivity from relativistic electrons (Rybicki \& Lightman 1979) which depends on $F_{e}(\gamma, t)$; the second term is

$$
\left[\frac{\partial}{\partial t} v(\varepsilon, t)\right]_{I C}=\frac{u_{\gamma}}{2 T} \int_{-1}^{1} d \mu(1-\mu) \int_{\gamma_{l}}^{\gamma_{\max }} d \gamma F_{e}(\gamma, t) \frac{\Phi\left(\varepsilon_{1}, t\right)}{\left\langle\gamma^{2}\right\rangle_{0}} \zeta \frac{\sigma_{K N}}{\sigma_{T}}
$$

where $\mu$ is the cosine of the scattering angle, $\gamma_{l}=\max \left(\gamma_{\min }, \varepsilon\right), \zeta=\gamma /(\gamma-\varepsilon)=1+2 \gamma(1-\mu) \varepsilon_{1}$, $\varepsilon_{1}=\varepsilon / 2 \gamma(1-\mu)(\gamma-\varepsilon)$, and $\sigma_{K N}=3 \sigma_{T}\left(\zeta^{2}-2 \zeta / 3+1\right) / 4 \zeta^{3}$ is the Klein-Nishina cross-section. By integrating both sides of equation (3) over all values of $\varepsilon$, we obtain $\left(d u_{\gamma} / d t\right)_{I C}$ on the left hand side, whereas on the right hand side we use the relation $\left|d \varepsilon / d \varepsilon_{1}\right|=2(1-\mu) \gamma^{2} / \zeta^{2}$ to convert the integral to be over $\varepsilon_{1}$ and integrate over all values of $\gamma, \varepsilon_{1}$, and $\mu$. Finally, we divide the result by 2 to avoid double counting of each scattering event, and obtain

$$
\left(\frac{d u_{\gamma}}{d t}\right)_{I C}=-\left(\frac{d u_{e}}{d t}\right)_{I C}=\frac{u_{\gamma}}{2 T} \int_{-1}^{1} d \mu \int_{\gamma_{\min }}^{\gamma_{\max }} d \gamma F_{e}(\gamma, t) \int_{\varepsilon_{\min }}^{\varepsilon_{\max }} d \varepsilon_{1} \Phi\left(\varepsilon_{1}, t\right) \frac{\gamma^{2}}{\left\langle\gamma^{2}\right\rangle_{0}}(1-\mu)^{2} \frac{1}{\zeta} \frac{\sigma_{K N}}{\sigma_{T}},
$$

where $\varepsilon_{\min , \max }$ are the limiting energies of photons in the plasma. In the Thomson regime $\zeta=1$ and $\sigma_{K N}=\sigma_{T}$, and one finds

$$
\left(\frac{d u_{\gamma}}{d t}\right)_{I C}=\frac{4 u_{\gamma}\left\langle\gamma^{2}\right\rangle}{3 T\left\langle\gamma^{2}\right\rangle_{0}}=\frac{4}{3} c n_{e} \sigma_{T} u_{\gamma}\left\langle\gamma^{2}\right\rangle
$$

in agreement with the well-known result for $\gamma \gg 1$ (Loeb et al. 1991). The coupled equations (1)-(4) are solved numerically for the radiation spectrum at the end of the cooling process (i.e., when $\left.u_{\gamma} \rightarrow u_{0}\right)$. Cooling ends after a relatively short time, $\lesssim 10 T \ll t_{0}$, for the parameters of interest here, and the photons decouple from the electrons subsequently. An example for the time evolution of the spectrum is shown in Figure 1a. It is evident that the radiation density above the $e^{+} e^{-}$-pair creation threshold is substantial.

The spectral evolution rate due to pair creation is described by

$$
\begin{aligned}
\frac{\partial}{\partial t}[v(\varepsilon, t)]_{ \pm} & =u_{\gamma}(t) \frac{\partial}{\partial t}[\Phi(\varepsilon, t)]_{ \pm}+\Phi(\varepsilon, t)\left(\frac{d u_{\gamma}}{d t}\right)_{ \pm} \\
& =\frac{u_{\gamma}}{T^{\prime}} \mathcal{I}_{1}(\varepsilon, t) \equiv-\frac{u_{\gamma}}{4 T^{\prime}} \int d \mu \int d \varepsilon^{\prime} \frac{\Phi(\varepsilon, t)}{\varepsilon} \frac{\Phi\left(\varepsilon^{\prime}, t\right)}{\varepsilon^{\prime}} \frac{\varepsilon}{\bar{\gamma}_{0}}(1-\mu) \frac{\sigma_{ \pm}}{\sigma_{T}}
\end{aligned}
$$


where the integration is over the range $-1 \leq \mu \leq 1$ and $\varepsilon_{\min } \leq \varepsilon \leq \varepsilon_{\max }$ subject to the condition $\varepsilon \varepsilon^{\prime}(1-\mu)>2$. Here $\sigma_{ \pm}$is the pair creation cross section (PS) and $1 / T^{\prime}=c n \sigma_{T} \bar{\gamma}_{0}^{2}$. In all examples considered in this Letter, we find that $t_{c} \mathcal{I}_{1}(\varepsilon, t) / T^{\prime} \ll 1$ for all relevant photon energies. Therefore, pair creation comes into play after the original electrons cool and decouple. The total energy loss per unit time and volume due to pair creation is obtained by integrating equation (6) over all values of $\varepsilon$,

$$
\left(\frac{d u_{\gamma}}{d t}\right)_{ \pm}=\frac{u_{\gamma}}{T^{\prime}} \int_{\varepsilon_{\min }}^{\varepsilon_{\max }} d \varepsilon \mathcal{I}_{1}(\varepsilon, t) \equiv \frac{u_{\gamma}}{T^{\prime}} \widetilde{\mathcal{I}}_{1}(t)
$$

The rate at which pairs are created per unit volume is

$$
\left(\frac{d n}{d t}\right)_{ \pm}=\frac{n}{T^{\prime}} \int_{\varepsilon_{\min }}^{\varepsilon_{\max }} d \varepsilon \mathcal{I}_{2}(\varepsilon, t) \equiv \frac{n}{T^{\prime}} \widetilde{\mathcal{I}}_{2}(t)
$$

where $\mathcal{I}_{2}(\varepsilon, t)$ is same as $\mathcal{I}_{1}(\varepsilon, t)$ except that $\varepsilon / \bar{\gamma}_{0}$ is absent in its integrand. At the beginning of pair creation, the energy density of radiation is $u_{0}$. The average Lorentz factor of the newly created pairs is $\bar{\gamma}_{ \pm}(t) \approx \bar{\gamma}_{0} \widetilde{\mathcal{I}}_{1}(t) / \widetilde{\mathcal{I}}_{2}(t)$, since $u_{\gamma} / n \approx \bar{\gamma}_{0}$. Because the pairs are born relativistic, they transfer almost all their energy to the radiation via IC scattering (and also synchrotron emission if $\zeta_{B} \approx \zeta_{e}$ ) on a time scale of order a few $T^{\prime}$. Similar cascades of pair creation and cooling occur in active galactic nuclei (Svensson 1987). Throughout the pair cascade and cooling process the radiation spectrum evolves continuously but its total energy density remains nearly constant at a value close to $u_{0}$. Figure $1 \mathrm{~b}$ shows an example of this evolution up to a time $\approx t_{0}$. Because the hydrodynamic time scale in the comoving frame $t_{\text {hyd }} \approx r / c \Gamma \approx 2 t_{0}$, pair processes can not continue to operate for times much longer than $t_{0}$ because of the decline in the densities due to the expansion of the fireball.

\section{Spectral Predictions of the Model}

We solved the coupled equations given above using the methods described by PS and obtained the model spectra for $E \approx 1.1 \times 10^{51} \mathrm{erg}, \bar{\gamma}_{p}=3, \zeta_{B}=0.1, \zeta_{e}=0.3$, and $p=3.5$. We assume a source redshift $z_{s} \approx 1$ and include its effect on photon energy and flux. The total observable fluence for the above parameters is $\approx 3.1 \times 10^{-7} \mathrm{erg} / \mathrm{cm}^{2}$.

Figure 1 shows the entire spectral evolution (in the comoving frame) due to the synchrotron and IC cooling and pair cascade processes. We take $M \approx 10^{27} \mathrm{~g}$ which yields $\Gamma \approx E / \bar{\gamma}_{p} M c^{2} \approx 400$ ( which are consistent with empirical constraints, e.g. Woods \& Loeb 1995); and a dissipation (or shell collision) radius of $3.3 \times 10^{13} \mathrm{~cm}$. Note that the assumed values for $E$ and $M$ correspond only to a single emitting shell; they should obviously be higher for the entire burst. For the above parameters, we get $\Delta \approx 4.1 \times 10^{10} \mathrm{~cm}, n \approx 1.1 \times 10^{12} \mathrm{~cm}^{-3}, \tau \approx 3 \times 10^{-2}$, and $B \approx 6.3 \times 10^{4} \mathrm{G}$. The characteristic time scale for synchrotron and IC cooling is $T=1.6 \times 10^{-5} t_{0}$. We find that after a time $t=t_{c} \approx 16 T$, only $10 \%$ of the initial energy remains with the electrons. Figure $1 \mathrm{a}$ illustrates that as a result of electron cooling, the location of the synchrotron peak shifts to lower energies and also becomes broader. Later on, another peak develops at a much higher energy due to IC losses, with KN suppression in the very high-energy tail. Figure 1b shows the evolution much later due to the pair-cascade process, which depletes the population of high-energy photons. 
The IC loss by these pairs produces a power law tail at yet higher energies. The low-energy synchrotron part of the spectrum is almost unaltered throughout this phase. The computation was stopped at $t \approx t_{0}$ at which time most of the photons leave the system.

Figure 2 shows the dependence of the emergent spectrum on the dissipation radius for a fixed value of $\Gamma$ (panel [a]) and dependence on $\Gamma$ for a fixed value of the dissipation radius (panel [b]). Panel (a) shows that the location of synchrotron peak moves up in energy as the radius decreases (with an opposite trend for the IC peak); hence the overall extent of the emission spectrum gets narrower at smaller radii. Since $B \propto n^{1 / 2} \propto r^{-3 / 2}$ and $\tau \propto r^{-2}$, as $r$ decreases the synchrotron frequency increases and the pair-production depletion of high-energy photons is enhanced. One of the model spectra in Figure (2a) is compared with the BATSE data through the empirical formula for the time-integrated flux given by Band et al. (1993 [B93]). In that case our model predicts $\sim 50 \%$ more time-integrated flux at $10 \mathrm{keV}$ than observed, which could be due to an oversimplified form of the electron distribution function which we have adopted. In our notation, the number of photons per unit interval of $\varepsilon$ is $d N / d \varepsilon \propto \varepsilon^{-1} \Phi(\varepsilon)$. Therefore, Band's formula (B93) reads

$$
\begin{aligned}
\varepsilon^{-1} \Phi(\varepsilon) & =c_{1} \varepsilon^{-\alpha} \exp \left(-\varepsilon / \varepsilon_{0}\right), \quad \text { if } \varepsilon \leq(\beta-\alpha) \varepsilon_{0} \\
& =c_{2} \varepsilon^{-\beta}, \quad \text { otherwise. }
\end{aligned}
$$

The constants $c_{1,2}$ are fixed by the requirement that this function be continuous at $\varepsilon=(\beta-\alpha) \varepsilon_{0}$ and the normalization $\int_{0}^{1} d \varepsilon \Phi(\varepsilon)=1$. Note that we have altered the signs of both indices $\alpha$ and $\beta$ relative to the convention of B93. The observed values of the parameters (cf. Table 4 in B93) are in the range $0.3<\alpha<1.5$ (although in a few cases $\alpha$ is zero or negative), $1.6<\beta<5$, and $15<\varepsilon_{0} m_{e} c^{2}(\mathrm{keV})<3 \times 10^{3}$. The majority of the bursts seem to have $\alpha \approx 1, \beta \approx 2$, and $\varepsilon_{0} m_{e} c^{2} \approx$ a few hundred keV. From the model spectra in Figure 2, it is clear that the emission extends over a wide range of photon energies, from the optical to the $\mathrm{TeV}$ regime. We expect more optical emission when the dissipation takes place at larger radii. It is very likely that internal shocks occur over a wide range of radii, thereby extending the spectrum to longer wavelengths, with simultaneous optical and x-ray emission during a GRB. Recent reports of nearly simultaneous detection of x-ray emission from GB960720 (Piro et al. 1997), GB970815 (Smith et al. 1997), and GB970828 (Remillard et al. 1997) are in qualitative agreement with our expectation for internal shocks.

\section{Conclusions}

We have shown that the emission spectra from internal shocks are affected by synchrotron emission, multiple Compton scatterings, and pair creation (Fig. 1). Our model spectra could mimic the observed BATSE spectra (B93) for reasonable choices of the shell Lorentz factor $\gamma \sim 10^{2}-10^{3}$ and shock radius $r \sim 10^{13}-10^{14} \mathrm{~cm}$ (Fig. 2).

The depletion of high energy photons due to pair creation is a sensitive probe of both $\Gamma$ 
and $r$. Detection of the high energy trough present in Figures (1b) and (2) can therefore be used to constrain these parameters. The potential degeneracy between the values of $\Gamma$ and $r$ can be removed on the basis of variability data, as the characteristic variability time scale depends on a different combination of these parameters, $\sim r / 2 \Gamma^{2}$. Despite their high-energy cut-off, our model spectra extend all the way up to photon energies as high as $10 \mathrm{GeV}-\mathrm{TeV}$.

We acknowledge discussions with M. Kamionkowski, T. Piran, M. A. Ruderman, and E. Woods. One of us (RP) thanks R. Sari for many critical comments and stimulating discussions during the VIII Marcel Grossmann meeting. This research was supported in part by NASA grants NAG5-618 and -2859 (for RP) and NASA ATP grant NAG 5-3085 and the Harvard Milton fund (for AL).

\section{REFERENCES}

Band, D. L. et al. 1993, ApJ 413, 281 (B93)

Bond, H. E. 1997, IAU Circ. No. 6654

Djorgovski, S. G. et al. 1997, Nature, 387, 786

Frail, D. A. et al. 1997, ApJ, 483, L91

Goodman, J. 1986, ApJ 308, L47

Hillas, A. M. 1984, Ann. Rev. Astron. Astrophys. 22, 425

Jun, B. and Norman, M. L. 1996, ApJ 472, 245

Kamionkowski, M. and Freese, K. 1992, Phys. Rev. Lett. 69, 2743

Kirk, J. G., in Plasma Astrophysics, edited by A. O. Benz \& T. J. L. Courvoisier, Saas-Fee Advanced Course 24 (Springer-Verlag, New York, 1994)

Kobayashi, S., Piran, T. \& Sari, R. 1997, astro-ph/970513 (KPS)

Loeb, A., McKee, C. F., \& Lahav, O. 1991, ApJ 374, 44

Mészáros, P. \& Rees, M. J. 1993, ApJ 405, 278 (MR)

Mészáros, P., Laguna, P., \& Rees, M. J. 1993, ApJ 415, 181

Metzger, M. R. et al. 1997, IAU Circ. No. 6655

Mignoli, M. et al. 1997, IAU Circ. No. 6661

Paczyński, B. 1986, ApJ 308, L43

Paczyński, B., \& Xu, G. 1994, ApJ, 427, 708

Pilla, R. P. \& Loeb, A. 1997, in preparation

Pilla, R. P. \& Shaham, J. 1997, ApJ 486, 903 (PS) 
Piran, T., Shemi, A., \& Narayan, R. 1993, MNRAS 263, 861 (PSN)

Piro, L. et al. 1997, A \& A, in press; astro-ph/9707215

Rees, M.J. \& Mészáros, P. 1994, ApJ 430, L93 (RM)

Remillard, R. et al. 1997, IAU Circ. No. 6726

Rybicki, G. B. \& Lightman, A. P. 1979, Radiative Processes in Astrophysics (New York: John Wiley)

Sari, R. \& Piran, T. 1997, MNRAS 287, 110 (SP)

Sari, R., Narayan, R., \& Piran, T. 1996, ApJ 473, 204 (SNP)

Smith, D. A. et al. 1997, IAU Circ. No. 6718

Svensson, R. 1987, MNRAS 227, 403

van Paradijs, J., et al. 1997, Nature, 386, 686

Vietri, M. 1997a, ApJ, 478, L9

-1997b, ApJ Letters, in press; astro-ph/9706060

Waxman, E. 1997a, ApJ Letters, in press; astro-ph/9704116 -1997b, astro-ph/9705229

Waxman, E. \& Piran, T. 1994, ApJ 433, L85

Wijers, A. M., Rees, M. J., \& Meszaros, P. 1997, MNRAS, 288, L51

Woods, E. \& Loeb, A. 1995, ApJ 453, 583

This preprint was prepared with the AAS LATEX macros v4.0. 




Fig. 1. - Time evolution of the radiation spectrum for $r=3.3 \times 10^{13} \mathrm{~cm}$; the values of all other parameters are specified in the text. Panel (a) shows the spectra at different times in the early phase of electron cooling where only synchrotron and IC cooling are effective. The normalized time and radiation density $\lambda=u_{\gamma}(t) / u_{0}$ are indicated for each curve. Because $\zeta_{B} / \zeta_{e}=0.33$, the IC component begins to grow only when $\lambda$ exceeds $\sim 0.3$, as expected from equation (1). Panel (b) shows the evolution much later due to pair creation. The final density of (cold) electrons and positrons is $\tilde{n} \approx 40 n$ in this example. However, they carry only a negligible fraction ( $\sim$ few percent) of the total energy. 


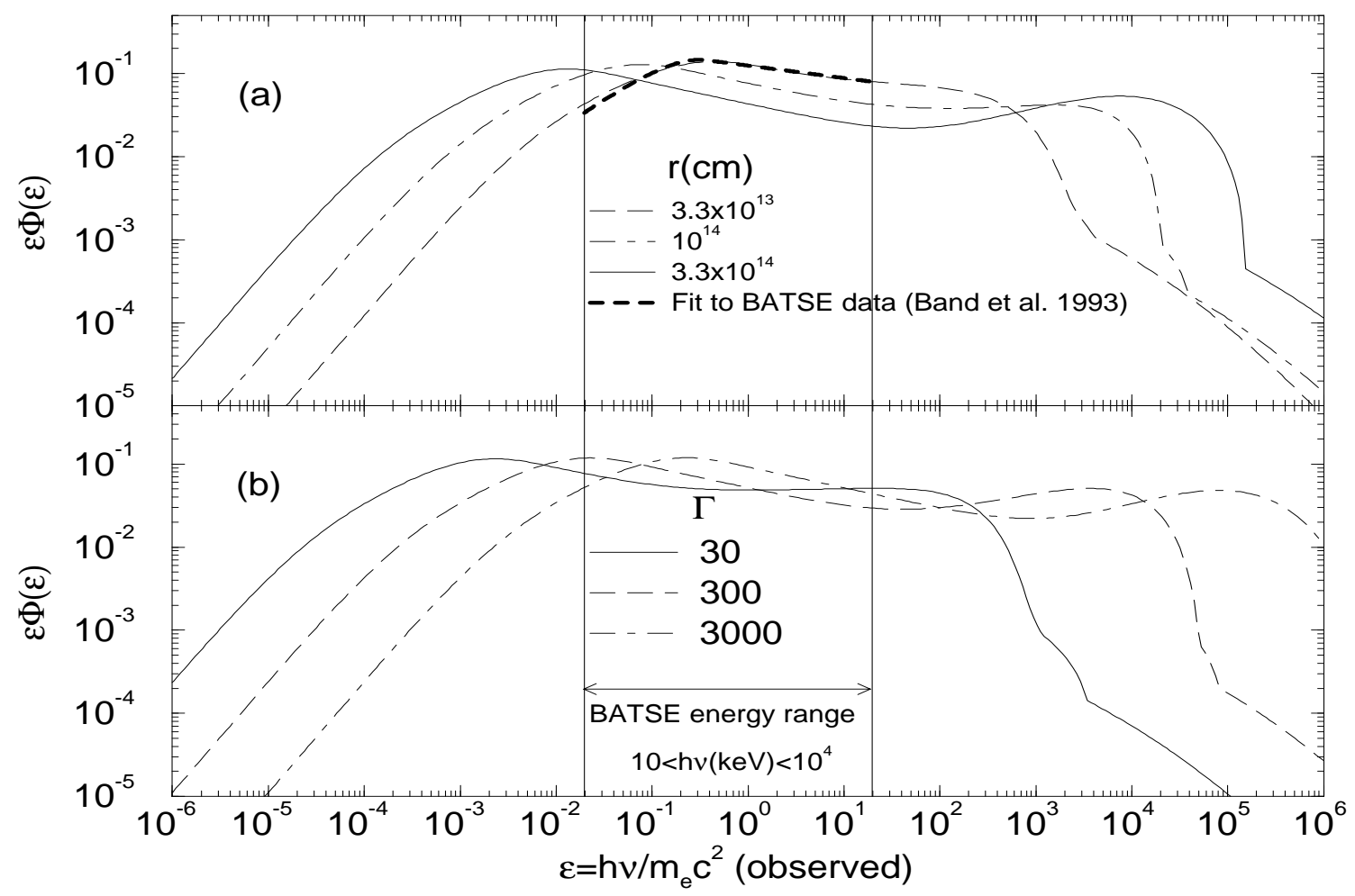

Fig. 2.- Dependence of the model spectra on the dissipation radius [panel (a)] and bulk Lorentz factor [panel (b)] of the emitting shell. On panel (a) we show also the Band spectrum [cf. Eq. (9)] for $\alpha=1.1, \beta=2.15$, and $\varepsilon_{0} m_{e} c^{2}=200 \mathrm{keV}$, which is in a good qualitative agreement with the predicted spectrum for $r=3.3 \times 10^{13} \mathrm{~cm}$. The vertical lines correspond to $10 \mathrm{keV}$ and $10 \mathrm{MeV}$, which roughly bracket the BATSE energy range. On panel (b) we show the $\Gamma$-dependence of the spectra for a fixed $r=2 \times 10^{14} \mathrm{~cm}$. The inferred emission radii and Lorentz factors are consistent with the empirical constraints set by the BATSE variability data (e.g., Woods \& Loeb 1995). 